\title{
Analytic computation of the energy levels of a two-dimensional hydrogenic donor in a constant magnetic field
}

\author{
Víctor M. Villalba *, and Ramiro Pino ${ }^{\dagger}$ \\ Centro de Física \\ Instituto Venezolano de Investigaciones Científicas, IVIC \\ Apdo 21827, Caracas 1020-A, Venezuela
}

\begin{abstract}
We compute the energy levels of a 2D Hydrogen atom when a constant magnetic field is applied. With the help of a mixed-basis variational method, we calculate the energy eigenvalues of the $1 S, 2 P^{-}$and $3 D^{-}$levels. We compare the computed energy spectra with those obtained via a generalization of the mesh point technique as well as the shifted $1 / N$ method. We show that the variational solutions present a good behavior in the weak and strong magnetic field regimes.
\end{abstract}

*e-mail:villalba@ivic.ivic.ve

†e-mail:rpino@ivic.ivic.ve 
The study of the behavior of hydrogenlike atoms in magnetic fields has been a subject of considerable interest in recent years. Stimulated by recent advances in nanofabrication technology that have made possible to create low dimensional structures containing one or more electrons: quantum wells, wires, and dots, a large body of papers 표 (and references therein) has considered the problem of two-dimensional donors in semiconductor superlattices. The most commonly studied semiconductor superlattice consists of regions of GaAs which act as wells for the conduction electrons separated by regions of $A l_{x} G a_{1-x} A s$ which act as barriers. Quantum wells in the presence of strong magnetic fields can form quantum dots, therefore the study of donor levels in $G a_{1-x} A l_{x} A s$ would be of help in understanding the role played by impurity ions in low dimensional structures. The computation of the binding energies as well as the study of exchange and correlation effects make it necessary to have at our disposal reliable analytic as well as numerical methods. In this direction, different approaches have been considered. Besides numeric and perturbation methods [5], we have to mention the two-point Padé approximation [8,g], as well as the shifted $1 / \mathrm{N}$ expansion [10,11.

The Hamiltonian describing the Coulomb interaction between a conduction electron and a donor impurity center when a constant magnetic field $\mathrm{B}$ is applied perpendicular to the $\mathrm{x}-\mathrm{y}$ plane can be written as

$$
H=-\nabla^{2}+\gamma L_{z}-\frac{2}{\rho}+\frac{\gamma^{2} \rho^{2}}{4}
$$

where we have chosen the vector potential $\mathbf{A}=\frac{B r}{2} \hat{e}_{\varphi}$ in the symmetric gauge. The coupling constant is $\gamma=\epsilon^{2} \hbar^{3} B /\left(c e^{3} m^{* 2}\right)$ where $m^{*}$ is the effective mass, $\epsilon$ is the dielectric constant of the host material. $L_{z}$ is the angular momentum operator $-i \hbar \partial / \partial \phi$ with eigenvalue $\hbar m$. The units of energy are given in terms of the effective Rydberg constant $\mathcal{R}_{0}^{*}=m^{*} e^{4} / 2 \hbar^{2} \epsilon^{2}$ and the effective Bohr radius $a^{*}=\hbar^{2} \epsilon / m^{*} e^{2}$, respectively.

The substitution

$$
\Psi=\frac{e^{i m \varphi}}{\sqrt{2 \pi}} \frac{u(\rho)}{\sqrt{\rho}}
$$

reduces the Schrödinger equation $H \Psi=E \Psi$ to the following second order ordinary differential equation

$$
\left[-\frac{d^{2}}{d \rho^{2}}+\left(m^{2}-\frac{1}{4}\right) \frac{1}{\rho^{2}}+\frac{\gamma^{2} \rho^{2}}{4}-\frac{2}{\rho}+m \gamma-E\right] u(\rho)=0
$$

It is well known that exact solutions of Eq. (3) cannot be expressed in closed form in terms of special functions [12]. There are analytical expressions for the energy for particular values of $\gamma$ and $m$ [13 [15]. In this paper, with the help of a mixed-basis variational method, we report a calculation of the $1 S, 2 P^{-}$and $3 D^{-}$energy levels of a $2 \mathrm{D}$ (x-y plane) hydrogenic atom in the presence of a strong magnetic field in the $\mathrm{z}$ direction; we compare our results with those obtained using the Schwartz [16] interpolation technique; as well as the shifted $1 / N$ method.

In order to apply the variational method to our problem [17], we look for a trial wave function. Since Eq. (3) reduces to the Hydrogen atom equation when $\gamma=0$, we can 
consider as a basis for $\gamma<<1$ the Hydrogen wave functions $\Psi_{H}$. Since $<\Psi_{H}|H| \Psi_{H}>\leq E$, we obtain an upper bound of the energy for small values of the parameter $\gamma$. The solution of Eq. (3) when $\gamma=0$ is

$$
u_{H}(\rho)=D_{m, n} e^{-\rho /\left(1 / 2+n_{\rho}+|m|\right)} \rho^{(|m|+1 / 2)} L\left(n_{\rho}, 2|m|, \frac{2 \rho}{\left(1 / 2+n_{\rho}+|m|\right)}\right)
$$

where $D_{m, n}$ is a normalization constant, and $\mathrm{L}(\mathrm{a}, \mathrm{b}, \mathrm{x})$ are the Laguerre polynomials [18]. Consequently the energy spectrum in the zero-field limit takes the form,

$$
E_{H}=-\frac{1}{\left(1 / 2+n_{\rho}+|m|\right)^{2}}
$$

Conversely, for large values of $\gamma$, a good trial basis is that of the spherical oscillator. In this case the solution of Eq. (3) has the form

$$
u_{O s c}(r)=C_{m, n} e^{-\gamma \rho^{2} / 4} \rho^{(|m|+1 / 2)} L\left(n_{\rho},|m|, \frac{\gamma}{2} \rho^{2}\right)
$$

and, in the high-field limit the energy levels are

$$
E_{O s c}=\gamma\left(2 n_{\rho}+|m|+m+1\right)
$$

If we attempt to apply the variational method using only the hydrogen atom basis, we will obtain a good agreement with the accurate results for small values of $\gamma$, but this approach fails for large $\gamma$ even if we consider a basis with many. Analogous situation occurs with the oscillator basis, for large $\gamma$, which converges very slowly for small values of $\gamma$. [7].

In order to solve this problem, we propose a mixed-basis approach. The idea is to use as trial function, for any quantum level $n$, a linear combination of the form

$$
u_{n}=\sum_{i}^{N}\left(c_{i H} u_{i H}+c_{N+i O} u_{i O s c}\right)
$$

where $N \geq i \geq n ; u_{i H}$ and $u_{i O s c}$ are the corresponding hydrogen and oscillator wave functions associated with the quantum level $i ; c_{i O}$ and $c_{i H}$ are constants to be calculated. It is worth noticing that our mixed basis is not orthogonal under the inner product $\int_{0}^{\infty} u_{i} u_{j} d \rho$. We proceed to minimize the expectation value $\langle u|H| u\rangle$ with the normalization condition, $\langle u \mid u\rangle=1$

After performing a variation on the basis coefficients $c_{i}$, we reduce our problem to that of solving the matrix equation

$$
\left[\left\langle u_{i}|H| u_{j}\right\rangle-\lambda\left\langle u_{i} \mid u_{j}\right\rangle\right] c_{j}=0
$$

after substituting the Hamiltonian (1) into (9), where the lowest value of $\lambda$ will be the energy of the level. When $j \leq 3$, we can analytically compute the energy eigenvalues $\lambda$ and eigenvectors $c_{j}$. The advantage of this approach is twofold. First, we have that the eigenvalues satisfy the inequality $\lambda \leq E$ and therefore we have a lower bound for our energy levels. Second, we obtain a relatively simple expression for the normalized eigenfunctions. 
In this paper we choose to work with a three term mixed-variational basis. In this case we can solve the resulting third-order algebraic equation (9) with the help of the Cardano method.

In order to compute the binding energy for the ground $1 S$ state, following the above proposed scheme, we have two possible trial functions

$$
u_{1 S}=c_{1} u_{1 S H}+c_{2} u_{1 S O}+c_{3} u_{2 S O} \quad(\operatorname{mix} 12)
$$

and

$$
u_{1 S}=c_{1} u_{1 S H}+c_{2} u_{2 S H}+c_{3} u_{1 S O} \quad(\text { mix } 21)
$$

It is worth mentioning that our three-term bases have the same angular dependence of the eigenfunction to be approximated. Analogously,

$u_{2 P^{-}}=c_{1} u_{2 P^{-} H}+c_{2} u_{2 P^{-} O}+c_{3} u_{3 P^{-} O}(\operatorname{mix} 12), u_{2 P^{-}}=c_{1} u_{2 P^{-} H}+c_{2} u_{3 P^{-} H}+c_{3} u_{2 P^{-} O}(\operatorname{mix} 21)$

and

$u_{3 D^{-}}=c_{1} u_{3 D^{-} H}+c_{2} u_{3 D^{-} O}+c_{3} u_{4 D^{-} O}(\operatorname{mix} 12), u_{3 D^{-}}=c_{1} u_{3 D^{-} H}+c_{2} u_{4 D^{-} H}+c_{3} u_{3 D^{-} O}(\operatorname{mix} 21)$

The numerical computations of the energy spectra associated with Eq. (3) will be carried out with the help of the Schwartz method [16] This method gives highly accurate results given a thoughtful choice of the reference function. For Eq. (3) we chose as the interpolation function

$$
f(\rho)=\sum_{m} f_{m} \frac{u(\rho)}{\left(\rho-r_{m}\right) a_{m}}
$$

with $u(\rho)=\sin \left[\pi(\rho / h)^{1 / 2}\right], r_{m}$ is a zero of $u(\rho), a_{m}$ is a zero of its derivative, and $h$ is the step of the quadratic mesh. The use of this scheme on Eq. (3) leads to an algebraic eigenvalue problem, giving as result a non-symmetric matrix to be diagonalized in order to obtain the energy values.

Here, as an illustration of the mixed-basis method, we present two different expansions of the $1 S, 2 P^{-}$, and $3 D^{-}$of the $2 \mathrm{D}$ Hydrogen Hamiltonian (1). We plot the energy against $\gamma^{\prime}=\gamma /(\gamma+1)$ as the horizontal scale. 


\section{FIGURES}

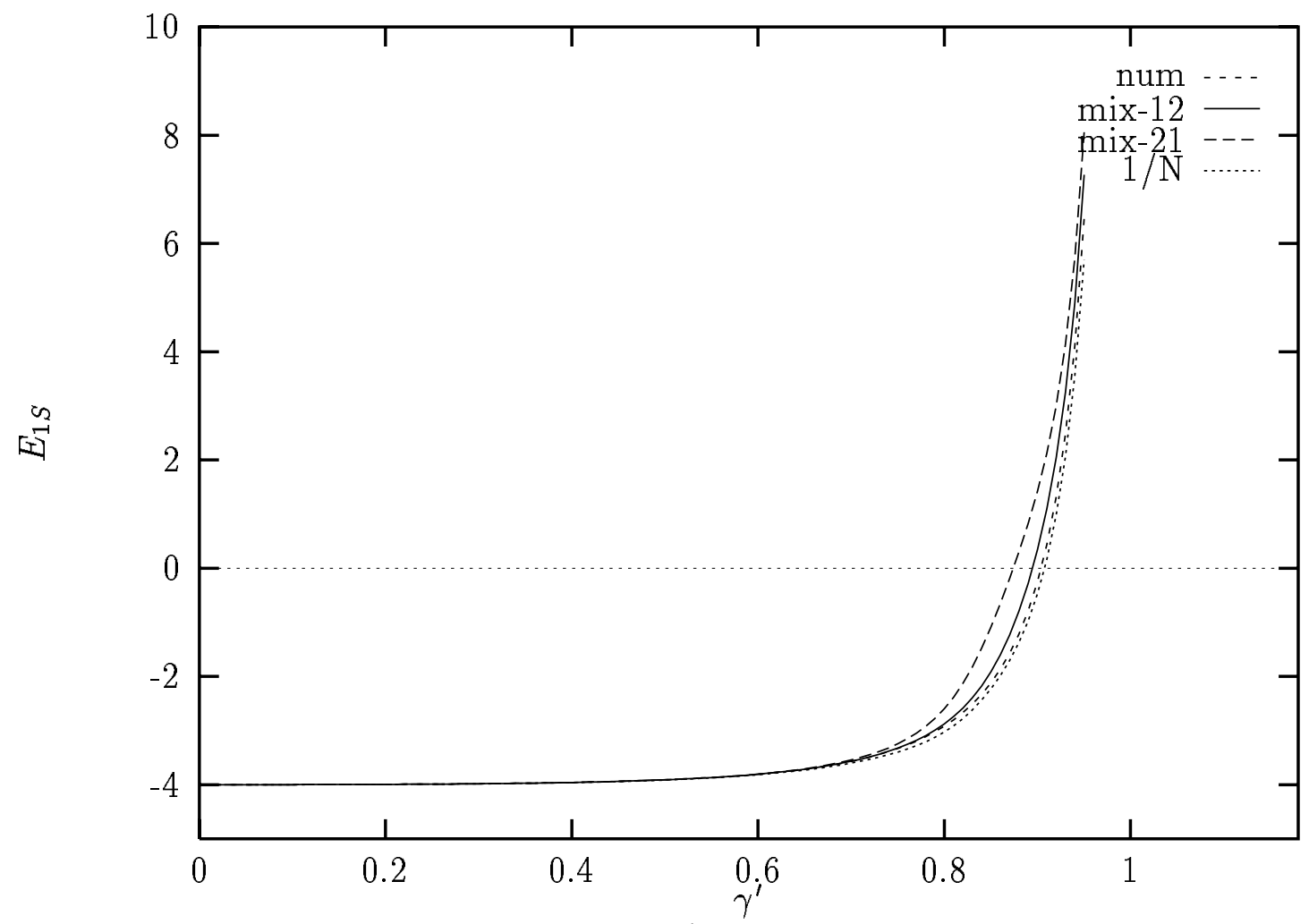

FIG. 1. Energy of the $1 S$ state as a function of $\gamma^{\prime}$. The light broken line is obtained by numerical methods; the dashed line corresponds to the mix 21 basis, (2S, 3S Hydrogen bases and 1S oscillator wavefunction). The solid line is obtained by using the mix12 basis (1S, $2 \mathrm{~S}$ oscillator bases and the $1 \mathrm{~S}$ Hydrogen wavefunction). The dotted line is obtained with the help of the shifted $1 / N$ method 


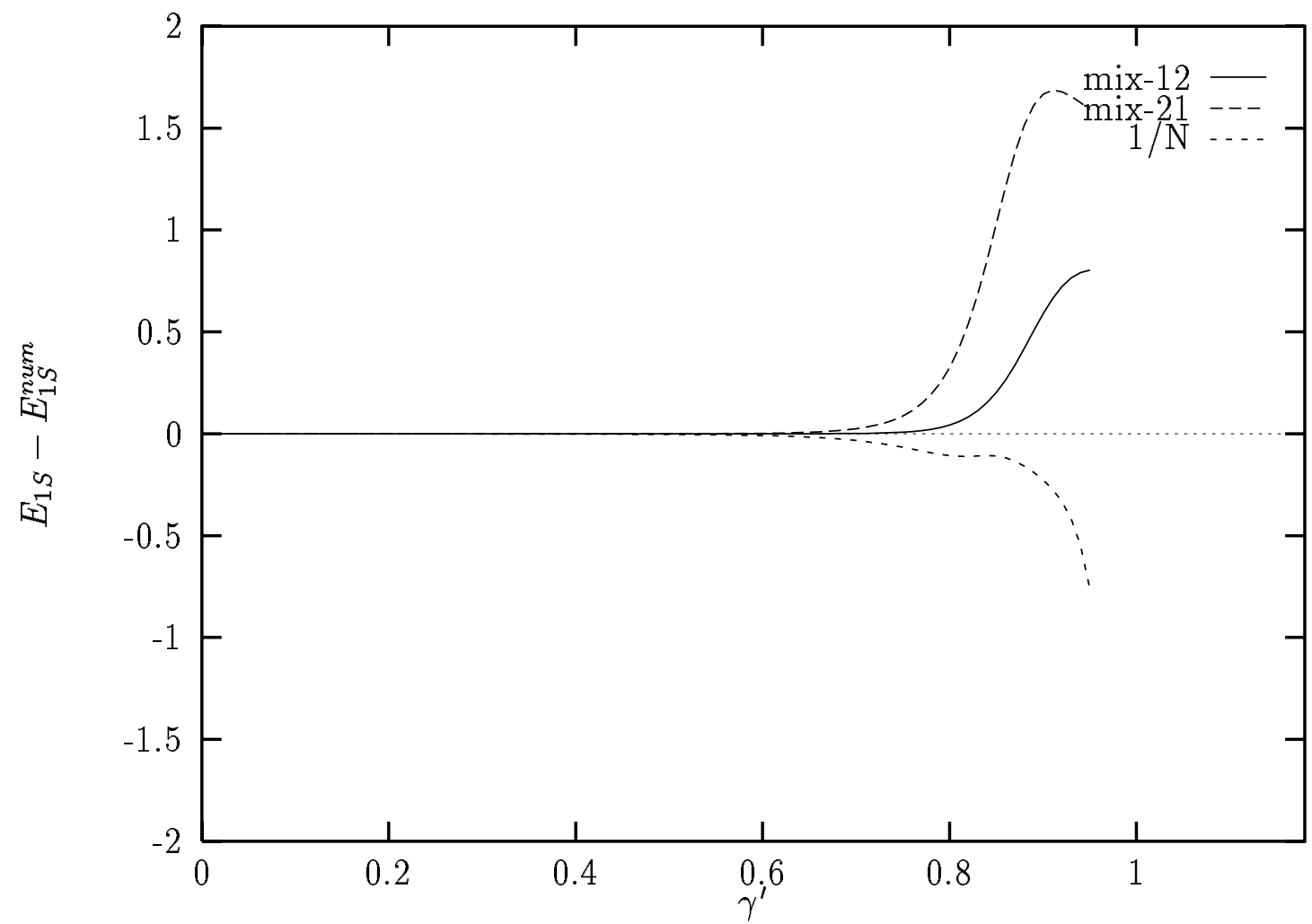

FIG. 2. The figure shows the difference between the numeric result for the $1 S$ energy spectrum and the energy values computed with the help of the mix12 basis (solid line), mix21 basis (heavy dashed line), and the shifted $1 / N$ method (light dashed line).

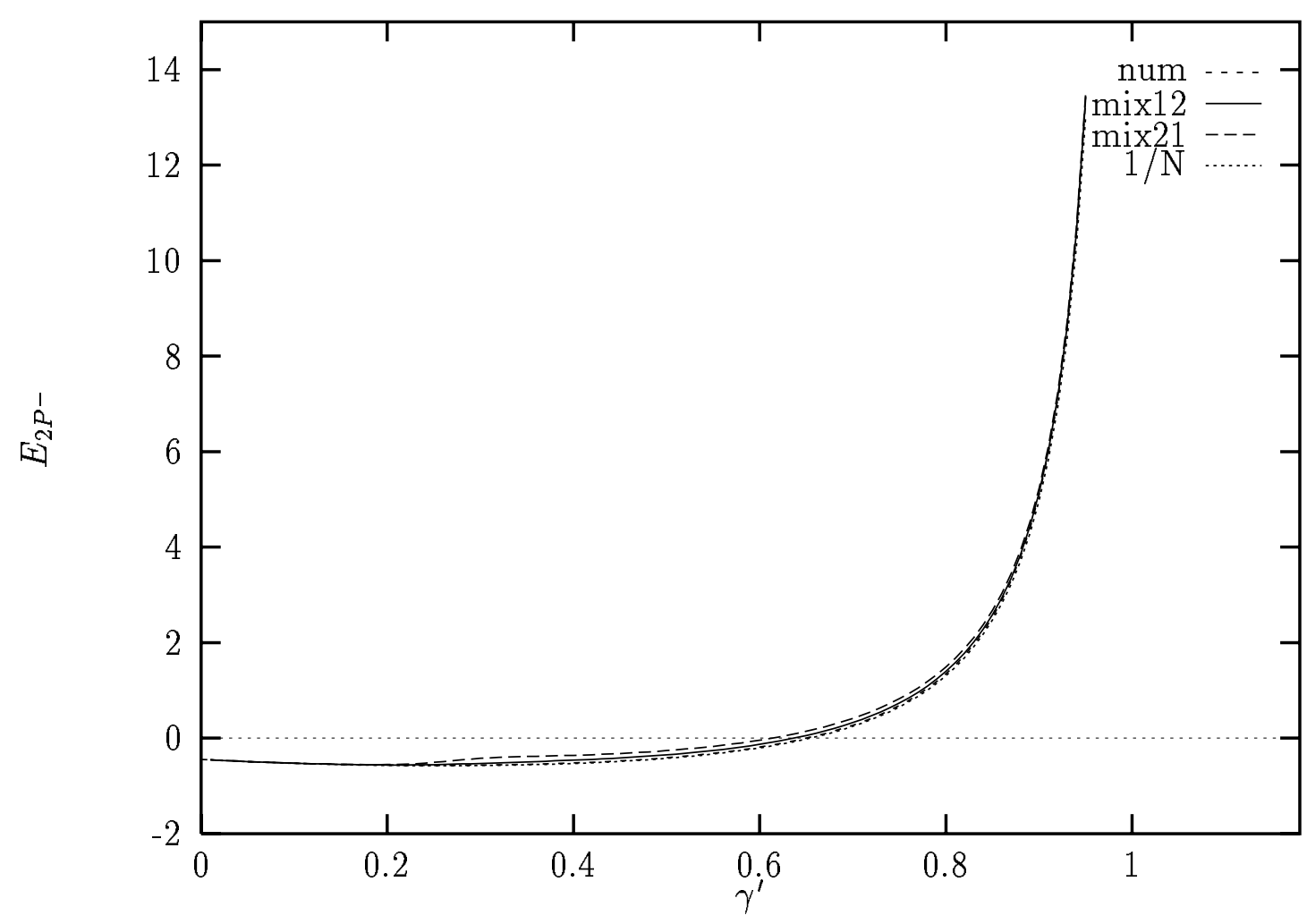


FIG. 3. Energy of the $2 P^{-}$state as a function of $\gamma^{\prime}$ The light broken line is obtained by numerical methods; the dashed line corresponds to the mix 21 basis, $\left(2 P^{-}, 3 P^{-}\right.$Hydrogen bases and $2 P^{-}$oscillator wavefunction). The solid line is obtained by using the mix 12 basis $\left(2 P^{-}, 3 P^{-}\right.$ oscillator bases and the $2 P^{-}$Hydrogen wavefunction). The dotted line is obtained with the help of the shifted $1 / N$ method.

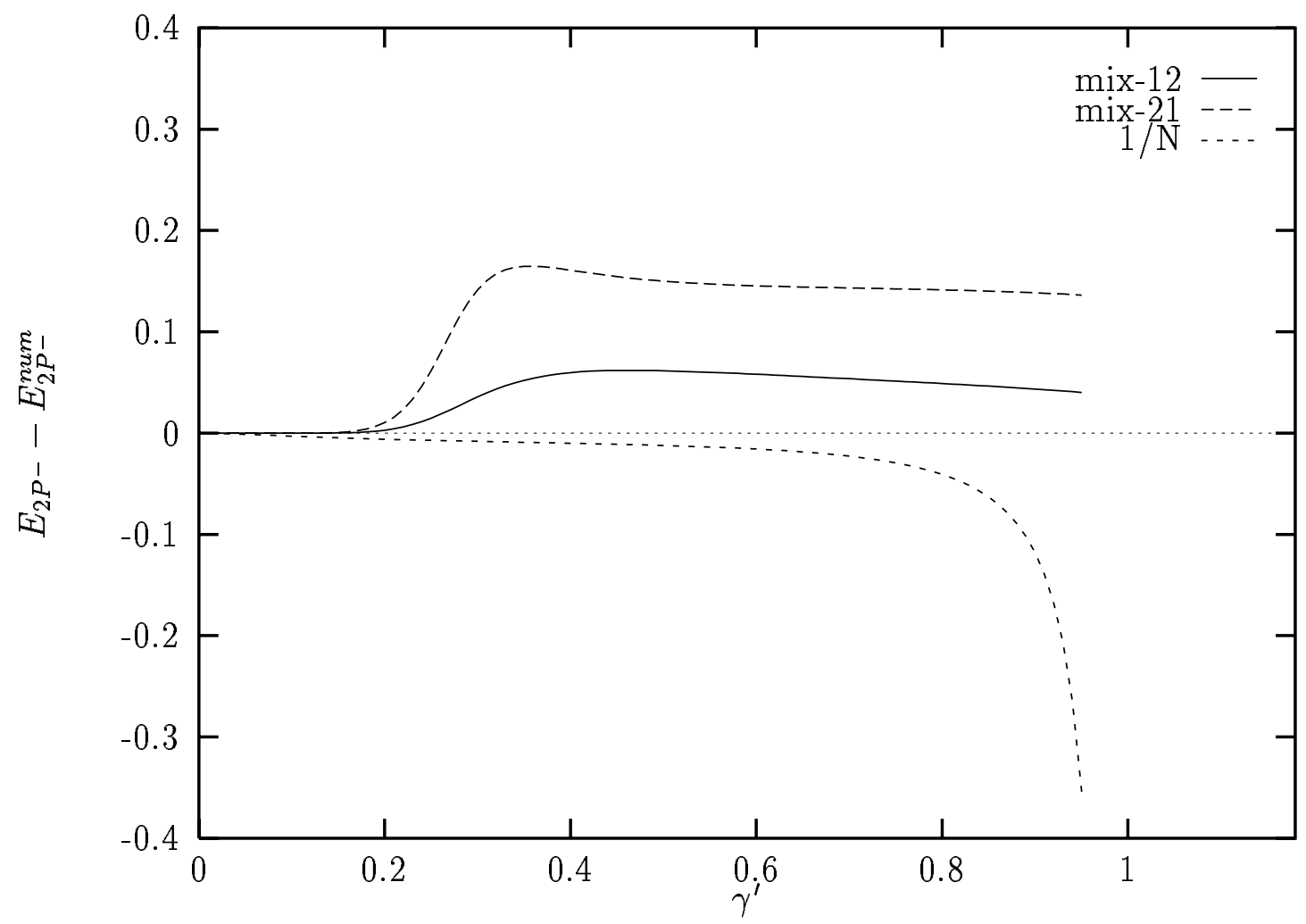

FIG. 4. The figure shows the difference between the numeric result for the $2 P^{-}$energy spectrum and the energy values computed with the help of the mix12 basis (solid line), mix21 basis (heavy dashed line), and the shifted $1 / N$ method (light dashed line). 


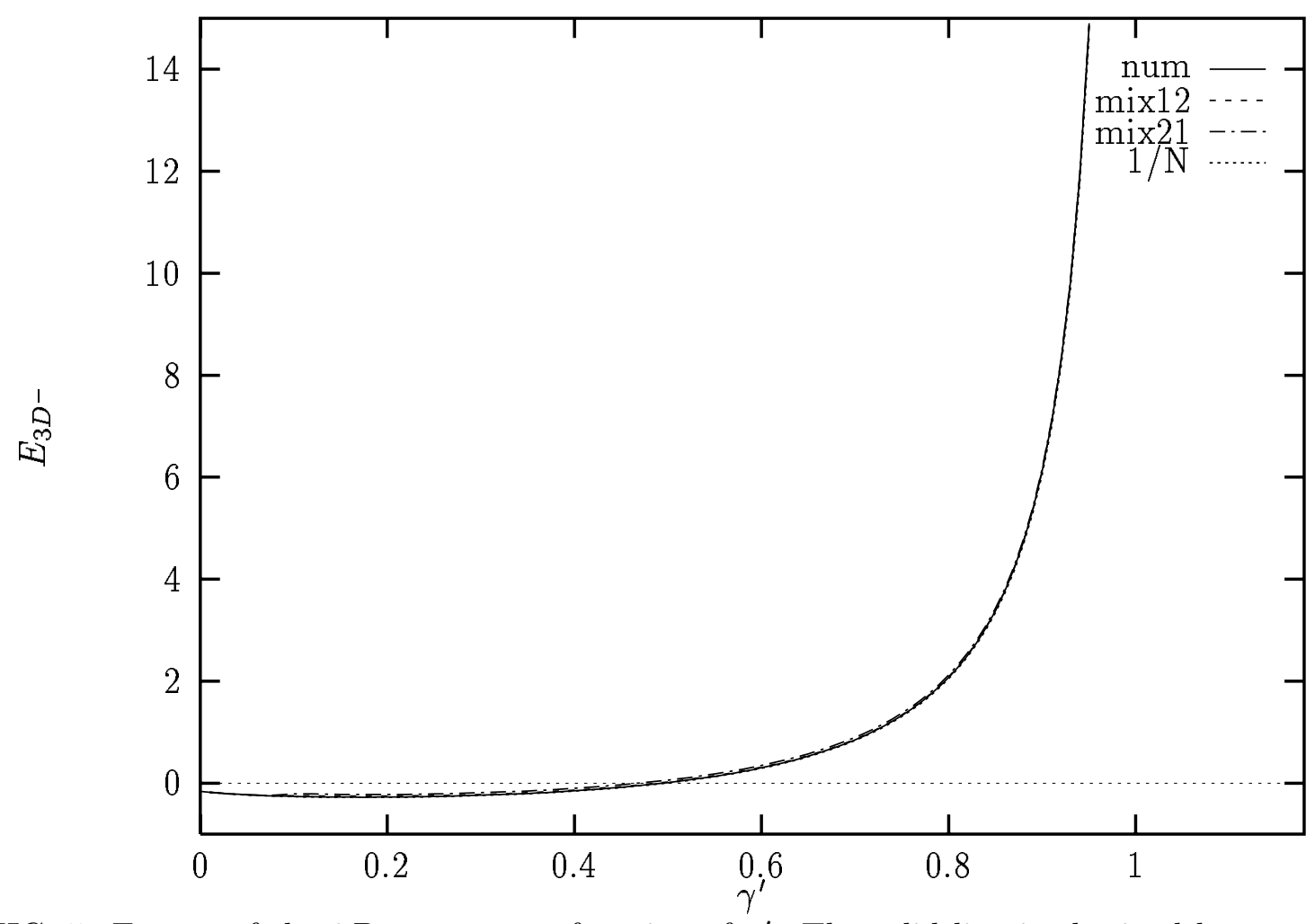

FIG. 5. Energy of the $3 D^{-}$state as a function of $\gamma^{\prime}$. The solid line is obtained by numerical methods; the heavy dashed line corresponds to the mix 21 basis $\left(3 D^{-}, 4 D^{-}\right.$Hydrogen bases and $3 D^{-}$ oscillator wavefunction). The light dashed line is obtained by using the mix12 basis $\left(3 D^{-}, 4 D^{-}\right.$ oscillator bases and the $3 D^{-}$Hydrogen wavefunction). The dotted line is obtained with the help of the shifted $1 / N$ method. 


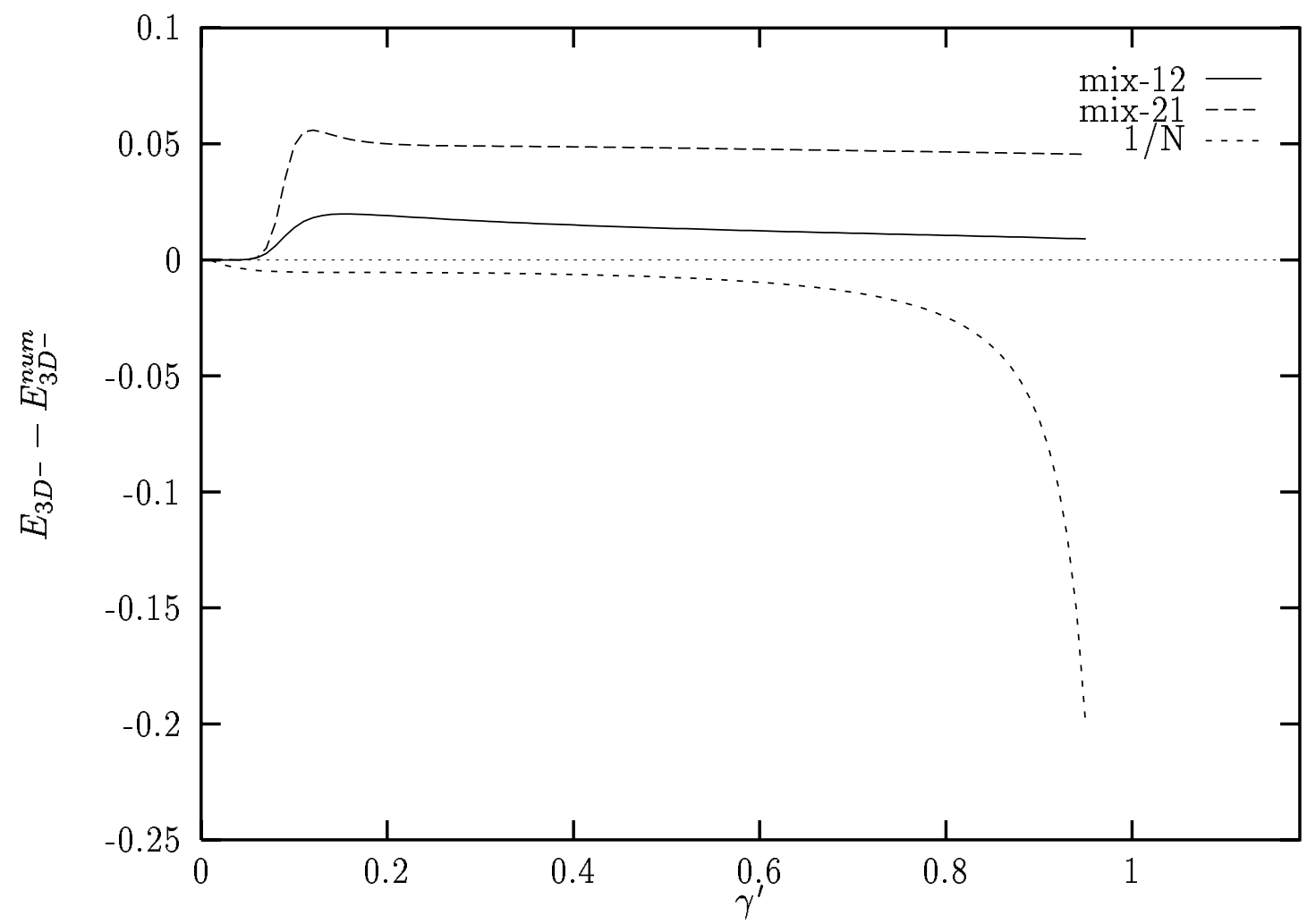

FIG. 6. The figure shows the difference between the numeric result for the $3 D^{-}$energy spectrum and the energy values computed with the help of the mix12 basis (solid line), mix21 basis (heavy dashed line), and the shifted $1 / N$ method (light dashed line).

It is easy to see that even for a two term mixed basis a good fit is obtained in the weak and strong field regimes. One of the basis gives a reasonably good fitting in the intermediate region. A better fit is obtained with the help of the mix12 bases. We also have that for the $2 P^{-}$and $3 D^{-}$states the mixed-basis variational approach gives very good results. Figures 1,3 , and 5 compare the variational results with those obtained numerically and with the help of the shifted $1 / N$ method.

It is difficult to decide on the evidence of Figs. 1, 3, and 5 which technique is better suited to the computation of the hydrogen energy levels. Figs. 2, 4, and 6 show that the shifted $1 / N$ method always gives results below the accurate energy levels. Although Figs. 1,3 , and 5 do not show mayor changes between the variational and shifted $1 / N$ method, the difference plots Figs. 2, 4, and 6 clearly show the improved performance of the mixed (mix12) variational method, especially for large values of $\gamma$.

It would be interesting to apply the mixed-basis technique for the 2D Hydrogen problem when relativistic effects are not negligible. This will be the object of a forthcoming publication.

\section{ACKNOWLEDGMENTS}

We thank Dr. Juan Rivero for helpful discussions. This work was supported by CONICIT under project 96000061 . 


\section{REFERENCES}

[1] Bastard, G., Phys. Rev. B 24, 4714 (1981)

[2] Greene, R. L., and Bajaj, K. K., Phys. Rev. B 31, 913 (1985)

[3] Zhu, J. L., Wu, J., Fu, R. T., Chen, H., and Kawazoe, Y. Phys. Rev. B 55, 1673 (1997)

[4] Sikorski, C. and Merkt, U., Phys. Rev. Lett 62, 2164 (1989)

[5] Whittaker, D. M. and Elliot, R. J. Solid State Commun. 68 , 1 (1988)

[6] Duggan, G. Phys. Rev B 37. 2759 (1988)

[7] Villalba, V. M. and Pino, R., J. Phys. Condens. Matter, 8, 8667 (1996)

[8] MacDonald, A. H. and Ritchie, D. S., Phys Rev B. 338336 (1986)

[9] Martín, P., Rodríguez, J. J. and Márquez, J. L. Phys. Rev. B. 45, 8359 (1992)

[10] Mustafa, O., J. Phys : Condens. Matter 5 , 1327 (1993)

[11] Mustafa, O., J. Phys. Condens. Matter 8, 8673 (1996)

[12] Bagrov, V. G, and Gitman D. M. Exact Solutions of Relativistic Wave Equations, (Kluwer, Dordretch, 1990)

[13] Lozanskii, E. D. and Firsov O. B., Izv. Vyssh. Ucheb. Zav. Fizika. 6, 52 (1974)

[14] Taut, M., J. Phys. A: Math. Gen. 28, 2081 (1995)

[15] Taut, M., Phys. Rev A. 48, 3561 (1993)

[16] Schwartz, C. J. Math. Phys, 26, 411 (1985)

[17] Davydov, A. S. Quantum Mechanics, (Pergamon, Oxford 1965)

[18] Lebedev, N. N., Special functions and their applications, (Dover, New York 1972) 